Gastroenterologe 2014 - 9:211-212

DOI 10.1007/s11377-014-0896-1

Online publiziert: 30. April 2014

(c) Springer-Verlag Berlin Heidelberg 2014

B. Kohler ${ }^{1}$ J.F. Riemann ${ }^{2}$

${ }^{1}$ Medizinische Klinik, Fürst-Stirum-Klinik, Bruchsal

2 em. Direktor der Med. Klinik C am Klinikum Ludwigshafen, c/o Stiftung LebensBlicke, Ludwigshafen

\title{
Komplikationsmanagement in der Endoskopie
}

auf das gesamte Spektrum aktueller therapeutischer Optionen wie Clipping, Stenting etc. ein.

Die ERCP kommt heute nahezu nur noch als therapeutisches Verfahren zum Einsatz. Diagnostische Fragestellungen werden durch alternative, weniger invasive Methoden wie EUS und MRCP beantwortet. Die Anzahl der retrograden Gangdarstellungen hat deshalb in der Vergangenheit drastisch abgenommen und begrenzt damit natürlich auch die Ausbildungsmöglichkeiten und Erfahrung unserer Assistenten; vielleicht ebenfalls ein Grund, warum die Komplikationsrate trotz verschiedener prophylaktischer Maßnahmen (NSAR rektal, Pankreasgangstenting) konstant bei ca. 10\% liegt.

Die Arbeit von Rabenstein und Schulz ist ein aktueller Überblick mit umfassendem Zahlenmaterial und vielen Details zu den Risikofaktoren und den 4 Hauptkomplikationen der ERCP (Pankreatitis, Blutung, Cholangitis, Perforation). der in etwa $10 \%$ der Fälle mit Komplikationen wie beispielsweise der Pankreatitis zu rechnen ist.

Die Ursachen von Komplikationen sind vielfältig, abhängig $u$. a. von der Art der endoskopischen Intervention, dem Krankheitszustand des Patienten, vor allem seinen Komorbiditäten, und natürlich der Erfahrung des Untersuchers. Komplikationen sind nicht immer zu vermeiden; von daher ist es umso wichtiger, sie frühzeitig zu erkennen und kompetent, u. a. interdisziplinär zu behandeln.

In der ersten Übersicht mit dem Titel „Perforation nach endoskopischen Eingriffen im Gastrointestinaltrakt" beschreiben Genthner et al. detailliert, umfassend und praxisnah die Vorgehensweise bei derartigen Komplikationen. Die Arbeit enthält viele Tipps und geht quasi

\section{) Speziell bei der Anasto- moseninsuffizienz ist die interdisziplinäre Zusammenarbeit absolut notwendig}

In einem weiteren Beitrag gehen Pauthner et al. auf die Probleme der postoperativen Anastomosenstenosen sowohl im oberen als auch im unteren Gastrointestinaltrakt ein. Sie geben hierbei wichtige Entscheidungstipps, wann diese Komplikation endoskopisch oder wann sie eher chirurgisch anzugehen sei. Neben der frühzeitigen Stentimplantation werden besonders die Erfahrungen mit der endoskopischen Vakuumschwammtherapie umfassend dargestellt. Speziell bei der Anasto- moseninsuffizienz ist die interdisziplinäre Zusammenarbeit absolut notwendig, als einem Paradebeispiel für ein gemeinsames viszeralmedizinisches Handeln.

Die Arbeit von Dormann und Jakobs fasst den aktuellen Stand des Stentings im Gastrointestinaltrakt, der Probleme und Komplikationen zusammen und bietet Lösungsmöglichkeiten bei schwierigen Situationen. Die Autoren beschreiben praxisnah und klinisch relevant die Vor- und Nachteile der unterschiedlichen Stents bei den verschiedenen Indikationen; momentan ist dies sicherlich einer der besten deutschsprachigen Artikel zu diesem Problem.

In der Übersicht zu Infektionen nach endoskopischen Eingriffen fasst M. Jung ausführlich die Risiken, die Möglichkeiten der Vorbeugung und die therapeutischen Maßnahmen zusammen, derartige Komplikationen zu vermeiden. Neben der Differenzierung der unterschiedlichen Infektionswege sowie deren Ursachen geht er ausführlich auf die neueren Empfehlungen zur Endokarditisprophylaxe ein - ein Paradigmenwechsel im Vergleich zu früheren Leitlinien. Gleichfalls von zunehmend klinischer Relevanz sind seine Ausführungen zum MRSA-Risiko und anderen multiresistenten Keimen in der Endoskopie.

Im letzten Beitrag stellen Riphaus und Wehrmann, die beide federführend an der Erstellung der S3-Leitlinie zur Sedierung in der Endoskopie beteiligt waren, neuere Studien zur Sedierung, insbesondere zur Propofol-Sedierung vor. Ergänzt wird der Artikel durch Empfehlungen zur Behandlung weiterer Komplikationen wie Hypertonie sowie Herzrhythmusstörungen.

Komplikationen in der Endoskopie sind erfreulicherweise selten, jedoch nicht 
immer zu verhindern, da manchmal methodenimmanent. Gleiches gilt für Komplikationen im Rahmen von operativen Maßnahmen am Gastrointestinaltrakt. Die frühzeitige Identifikation der Situation und ein professionelles Komplikationsmanagement sind die unabdingbare Voraussetzung für die erfolgreiche Beherrschung derartiger Zwischenfälle. Zur Aus- und Weiterbildung eines jeden Endoskopikers muss heute daher zwangsläufig auch das Komplikationsmanagement gehören.

Die Herausgeber hoffen, dass das vorliegende Schwerpunktheft dem interessierten Leser hilft, Komplikationen zu vermeiden, sowie eine Anleitung darstellt, diese auch fachgerecht zu diagnostizieren und zu behandeln.

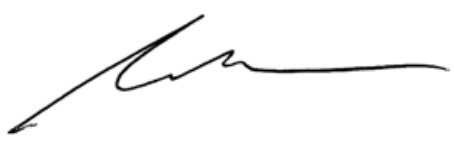

Prof. Dr. B. Kohler

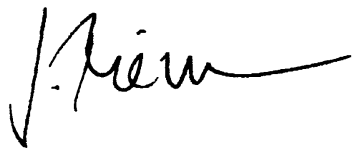

Prof. Dr. J.F. Riemann

\section{Korrespondenzadressen

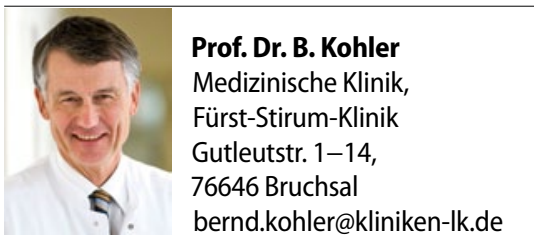

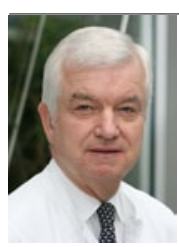

Prof. Dr. J.F. Riemann em. Direktor der Med. Klinik C am Klinikum Ludwigshafen, c/o Stiftung LebensBlicke Schuckertstr. 37, 67063 Ludwigshafen riemannj@garps.de

\section{Labordiagnostik braucht internistische Kompetenz}

Klinische Bewertung von Laborbefunden muss abrechenbar bleiben / Warnung vor völliger Abkopp-

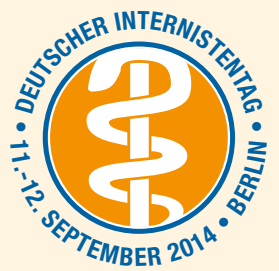
lung von Labor und internistischer Tätigkeit

In weiten Teilen der Inneren Medizin sind die korrekte Anforderung und sachkundige Interpretation von Laborparametern eine Grundvoraussetzung für eine qualitativ hochwertige, leitliniengerechte Patientenversorgung. So ergeben sich unter anderem in der Endokrinologie und der Rheumatologie aus den oft sehr umfangreichen Laborpanels für Hormone bzw. Entzündungsparameter unmittelbare therapeutische Konsequenzen.

Für die Patienten ist es dabei essentiell, dass die Laborparameter vom behandelnden Arzt korrekt bewertet und eingeordnet werden. Im Einzelfall kann das sehr komplex sein, weil Laborwerte interagieren können und ihre korrekte Interpretation unter anderem vom Alter, der Medikation und den Begleiterkrankungen des Patienten abhängt. „Leider ist in letzter Zeit zu beobachten, dass sowohl in der Musterweiterbildungsordnung als auch in den Vergütungssystematiken die für die Interpretation von Laborwerten nötige Fachkompetenz nicht mehr ausreichend berücksichtigt wird", betont Dr. med. H.-F. Spies, der 2. Vizepräsident des Berufsverband Deutscher Internisten e.V. (BDI).

Beim 7. Deutschen Internistentag in Berlin hat der BDI deswegen eine berufspolitische Podiumsdiskussion unter dem Titel "Gehört das Labor in die Innere Medizin?" ins Programm genommen. Unter der Leitung von Professor Dr. U. Fölsch vom BDI diskutieren die BDI-Expertin für Endokrinologie Dr. C. Jaursch-Hancke, Dr. M. Müller vom Berufsverband Deutscher Laborärzte, außerdem Dr. E. Edelmann vom Berufsverbands der Deutschen Rheumatologen sowie Dr. G. von Knoblauch von der Landesärztekammer
Hessen, Prof. Dr. H. Rebscher von der DAKGesundheit und Dr. D. Auch von der Kassenärztlichen Bundesvereinigung.

„Aus Sicht des Internisten sehen wir die zunehmende Abkopplung des Labors von der ärztlichen Tätigkeit außerordentlich kritisch", betont Spies. Dabei geht es nicht nur darum, wieder eine adäquate Abrechenbarkeit der Interpretation von Laborwerten zu erreichen, etwa durch eine Splittung der Vergütung oder eine Aufwertung der internistischen Bewertung des Labors. „Es geht uns auch darum, dass Internisten im Rahmen von Kooperationsstrukturen weiterhin wie in der Vergangenheit selbst Laborleistungen qualitätsgesichert erbringen können."

Wird die derzeitige Laborpolitik von den an der Aushandlung der Vergütungsmodelle beteiligten Verbänden weiter verfolgt, drohen nicht nur einschneidende Veränderungen für freiberufliche Ärzte in internistischen Schwerpunktpraxen. „Letztlich gehen die aktuellen Entwicklungen auch gegen die Interessen der Labormediziner, die Gefahr laufen, über kurz oder lang ihren Status als ärztliches Fachgebiet zu verlieren", betont Spies.

\footnotetext{
จ Veranstaltung "Gehört das Labor in die Innere Medizin? Qualitätsvorgaben und Mengenentwicklung" am Do., 11. September 2014 um 15.15 Uhr im Raum Langenbeck des LangenbeckVirchow-Hauses Berlin.
}

Mehr unter:

www.internistentag.de 